\title{
Cardio Metabolic Overrun in A Context of Covid 19: A Real Problem of Care in Sub-Saharian Africa
}

\author{
Diop KR ${ }^{1 *}$, Beye SM2, Mingou JS ${ }^{1}$, Diouf $Y^{1}$, Ndiaye $M^{3}$, Diop CMBM ${ }^{1}$, Ndiaye PG $^{1}$, Diallo $S D^{1}$, Akanni $S^{1}$, Sarr \\ $\mathrm{SA}^{1}$, Fatou $\mathrm{AW}^{1}$, Bodian $\mathbf{M}^{1}$, Ndiaye $\mathrm{MB}^{1}$, Kane $\mathrm{AD}^{1,2}$, Diao $\mathbf{M}^{1}$, Kane $\mathrm{A}^{3}$ and Pouye $\mathrm{A}^{4}$
}

${ }^{1}$ Department of Cardiology, Aristide Le Dantec Hospital, Senegal

${ }^{2}$ Department of Cardiology, Regional Hospital of Saint Louis, Senegal

${ }^{3}$ Department of Cardiology, Dallal Jamm Hospital, Senegal

${ }^{4}$ Clinique Medicale 1 Aristide Le Dantec Hospital, Senegal

*Corresponding author: Diop KR, Department of Cardiology, Aristide Le Dantec Hospital, Dakar, Senegal.

Received Date: October 01, 2020

Published Date: November 12, 2020

\begin{abstract}
Introduction: Coronavirus disease (COVID-19) is a real public health problem around the world. The first case was reported in Wuhan, China in December 2019.

Case presentation: It was a 31-year-old patient without cardiovascular risk factors and pathological history. He was hospitalized in an Epidemic Treatment Center (ETC) for an asymptomatic infection with SARS-CoV2 (Acute Severe Respiratory Syndrome Coronavirus 2). On the sixth day of his hospitalization, He developed inaugural decompensated diabetes under keto-acidosis mode, successfully treated by insuline and rehydratation. A week later, he presented a typical chest pain, during rest. The hemodynamic was stable, the physical examination found an irregular tachycardia. Standard electrocardiogram (ECG) showed ST elevation on anterior leads and atrial flutter with variable conduction $(2 / 1,3 / 1)$. In this context of COVID 19 in ETC, there was no possibilities for carrying out additional examinations: transthoracic echocardiography, coronary angiography or magnetic resonance imaging. We have in this context of typical anginal pain and electrical abnormalities, considered as an acute coronary syndrome with elevated and a pharmacological revascularization strategy has been initiated without success. The atrial flutter was successfully traited by a biphasic cardioversion in a context of hemodynamic instability.
\end{abstract}

Conclusion: COVID-19 interacts with the cardiovascular system on multiple levels, increasing morbidity in patients with underlying cardiovascular disease and causing myocardial damage and dysfunction

Keywords: COVID 19; Young patient; Cardiac decompensation; Sub-Saharian Africa

\section{Introduction}

Coronavirus disease (COVID-19) is a real public health problem around the world. The first case was reported in Wuhan, China in December 2019 [1]. There are 6.2 millions confirmed cases of COVID-19 around the world, with more than 375,000 deaths [2]. COVID-19 has become a global pandemic and has mobilized health professionals from all disciplines [3]. We report the case of a young patient without cardiovascular risk factors or comorbidities, He was admitted for an asymptomatic case of COVID-19. He presented during hospitalization in an Epidemic Treatment Center (ETC), an inaugural diabetes and cardiac decompensation (Acute Coronary Syndrome and atrial flutter). In the follow-up in the ETC, there were limitations in the paraclinical exploration of the patient, as neither an echocardiography or a coronary angiography could be performed due to COVID 19. 


\section{Case Presentation}

It was a 31-year-old patient without cardiovascular risk factors and pathological history. He was hospitalized in the ECT for an asymptomatic infection with SARS-CoV2 (Acute Severe Respiratory Syndrome Coronavirus 2). He was treated with Azithromycin and Hydroxychloroquine. On the sixth day of his hospitalization, he developed inaugural decompensated diabetes under keto-acidosis mode. An insulin therapy and a parenteral rehydration had been started. The evolution was marked by a stabilization of glycemia. A week later, he presented a typical chest pain, at rest. The hemodynamic was stable, Physical examination found an irregular tachycardia. Standard electrocardiogram (ECG) performed showed an ST elevation on the anterior leads and atrial flutter with variable conduction $(2 / 1,3 / 1)$ with a ventricular rate of 150 cycles / minute
(Figure 1). There was a non specific biological inflammatory syndrome and an elevation of Troponin to 100 times normal. In this context of COVID 19 in ETC, there was no possibilities for carrying out additional examinations: echocardiography, coronary angiography or magnetic resonance imaging.

We have in this context of typical chest pain, electrical abnormalities, and troponine elevation, considered as an ST elevation myocardial infarctus (STEMI) and a pharmacological revascularization strategy has been initiated with stroptokinase without success (Figure 2). He recieved $5 \mathrm{mg}$ of bisoprolol to control the heart rate. Ragards to hemodynamic instability, with the persistance of the flutter desspite that introduction of bisoprolol, successful cardioversion was obtein after 100 joules of biophasic Choc (Figure 3).

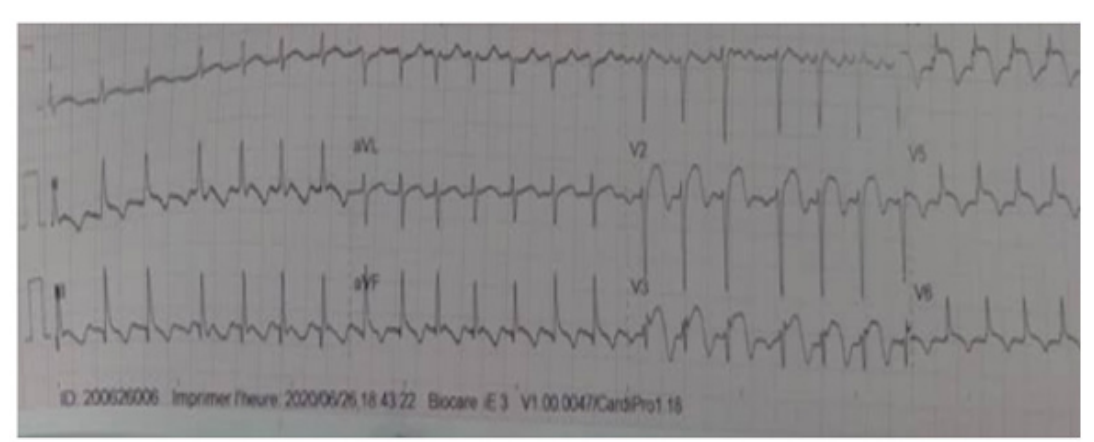

Figure 1: ECG showing the anterior ST segment elevation and variable conduction atrial flutter $(2 / 1,3 / 1)$ with a ventricular rate of 150 cycles $/ \min$

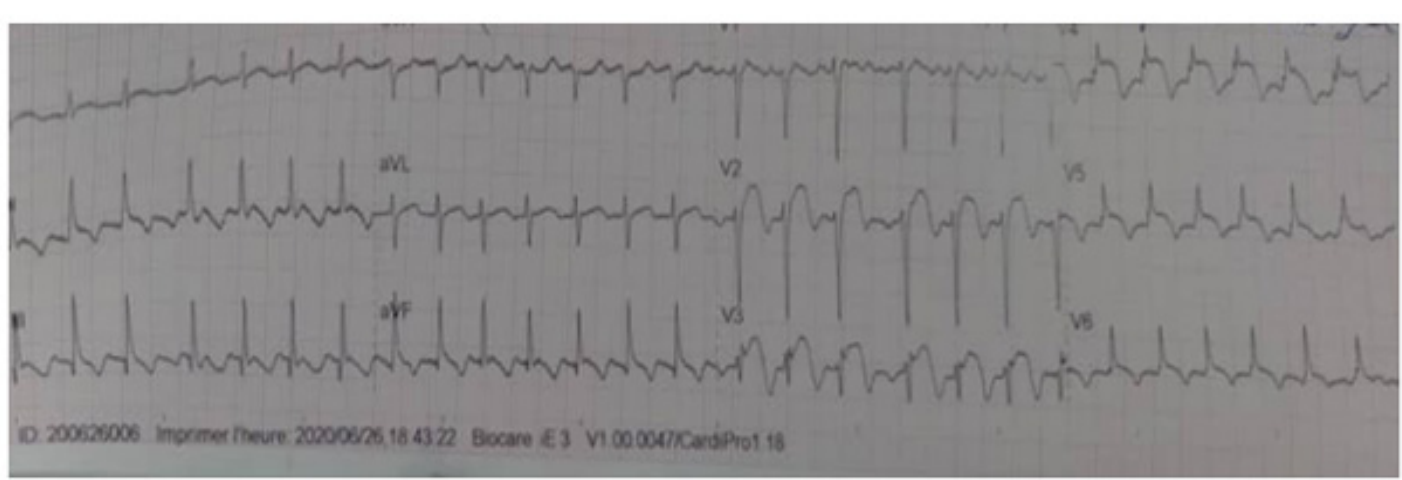

Figure 2: ECG performed after thrombolysis.

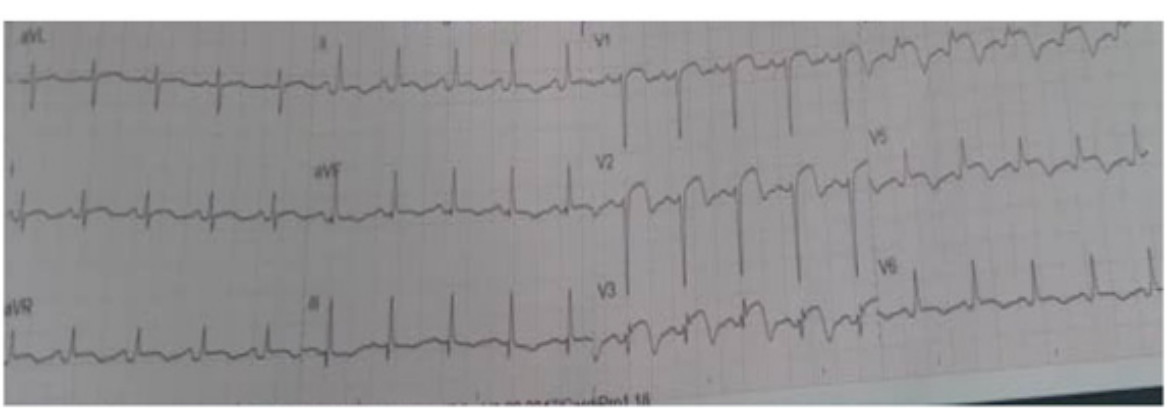

Figure 3: ECG performed after electrical cardioversion: showing a sinus heart rate with a frequency of $110 \mathrm{cycles} / \mathrm{min}$. 


\section{Discussion}

COVID-19 has become a global pandemic and has mobilized health professionals from all disciplines [3]. The clinical profile varies from the asymptomatic form, through the mild or moderate forms to severe or even critical forms with multivisceral dysfunction leading to the onset of diabetes, cardiovascular involvement among others [3]. Cardiovascular lesions, often evidenced by elevated cardiac biomarkers, were recognized among the first cases in China [4]. These lesions are not uncommon with COVID-19 disease and their presence portends both more serious illness and higher mortality [3]. The exact mechanism of cardiac involvement in COVID-19 remains under investigation. One potential mechanism is the direct involvement of the myocardium through angiotensin converting enzyme 2 receptors [5]. Other mechanisms suggesting cardiac involvement linked to COVID-19 include a cytokine storm, mediated by an unbalanced immune response [6]. Our clinical case deserves special attention because it is about a young subject without comorbidities, who presented an inaugural diabetes and a chest pain associated with an STEMI. We were confronted with a real diagnostic and support problem due to the lack of investigative equipement (echocardiography or angiography, MRI) inside the ECT. An other problem was the absence of a circuit covid clearly identified to do these investigations, even though these equipments are available in the hospital but could not receive this patient.

A myocarditis, or Tako-tsubo could not be ruled out or retained, as no exploration capable of supporting these diagnoses could be performed in our context. The interest of this case is to show the difficulties of diagnostic management in front of the absence of a circuit for performing a transthoracic echocardiography, coronary angiography or magnetic resonance imaging, in context of COVID-19 in Senegal.

\section{Conclusion}

This cardio-metabolic damage occurance in a patient with covid19 is very uncommon. COVID-19 interacts with the cardiovascular system on multiple levels, increasing morbidity in patients with underlying cardiovascular disease and causing myocardial damage and dysfunction. It is undoubtedly necessary to develope a course of care within each cardio-metabolic institution to organize our approach to the problems linked to the cardiovascular complications of COVID-19.

\section{Acknowledgement}

None.

\section{Conflict of Interest}

No conflict of interest.

\section{References}

1. Bo Li, Jing Yang, Faming Zhao, Lili Zhi, Xiqian Wang, et al. (2020) Prevalence and impact of cardiovascular metabolic diseases on COVID-19 in China. Clin Res Cardiol 109(5): 531-538.

2. Tao Guo, Yongzhen Fan, Ming Chen, Xiaoyan Wu, Lin Zhang, et al. (2020) Cardiovascular Implications of Fatal Outcomes of Patients With Coronavirus Disease 2019 (COVID-19). JAMA Cardiol 5(7): 811-818.

3. Judson GL, Kelemen BW, Njoroge JN, Vaikom S Mahadevan (2020) Cardiovascular Implications and Therapeutic Considerations in COVID-19 Infection. Cardiol Ther 9(2): 293-305.

4. Kevin J Clerkin, Justin A Fried, Jayant Raikhelkar, Gabriel Sayer, Jan M Griffin, et al. (2020) COVID-19 and Cardiovascular Disease. Circulation 141(20): 1648-1655

5. GY Oudit, Z Kassiri, C Jiang, PP Liu, SM Poutanen, et al. (2009) SARS-coronavirus modulation of myocardial ACE2 expression and inflammation in patients with SARS. Eur J Clin Invest 39(7): 618-625.

6. Ying-Ying Zheng, Yi-Tong Ma, Jin-Ying Zhang, Xiang Xie (2020) COVID-19 and the cardiovascular system. Nat Rev Cardiol 17(5): 259-260. 